\title{
Distribución y estado poblacional del lobo de río (Pteronura brasiliensis) en la cuenca del río Madre de Dios, sureste del Perú
}

\section{Giant Otter (Pteronura brasiliensis) distribution and population status in Madre de Dios River basin, southeastern Peru}

\author{
Joel Alexander Mendoza ${ }^{1 *}$, Keyly Huamani ${ }^{1}$, Germán Sebastián ${ }^{1}$ y José Antonio Ochoa ${ }^{1,2}$ \\ 1 Frankfurt Zoological Society - Perú, Residencial Huancaro, Bellavista M-1, Santiago, Cusco, Perú. \\ 2 Universidad Nacional de San Antonio Abad del Cusco, Museo de Historia Natural. Paraninfo Universitario, Plaza de Armas s/n, Cusco, Perú. \\ * Autor para correspondencia \\ Email: Joel A. Mendoza: joel.mendoza@fzs.org \\ Email: Keyly Huamani: keyly.huamani@fzs.org \\ Email: Germán Sebastián: german.sebastian@fzs.org \\ Email José A. Ochoa: jose.ochoa@fzs.org
}

\begin{abstract}
Resumen
Se presenta información del estado poblacional y distribución geográfica del lobo de río (Pteronura brasiliensis) en la región Madre de Dios durante los años 2014 y 2015. La población registrada fue de 128 individuos para ambos años, agrupados en 22 grupos familiares en el año 2014 y 20 grupos en el año 2015. Los sectores con mayores abundancias fueron el Parque Nacional del Manu con 2.2 ind/10 km, seguido del Parque Nacional Bahuaja Sonene con 1.43 ind/10 km. La mayor población de lobo de río fuera de las áreas naturales protegidas (ANP) fue registrada en la cuenca del río Las Piedras con una abundancia de $0.5 \mathrm{ind} / 10 \mathrm{~km}$, seguido de la cuenca de Los Amigos con $0.43 \mathrm{ind} / 10 \mathrm{~km}$. Las poblaciones dentro de ANP se muestran estables y protegidas (17 grupos familiares en ambos años), mientras que fuera de ANP se registraron solamente 5 grupos en el año 2014 y 2 grupos en el 2015. Actualmente, las mayores amenazadas para las poblaciones de lobo de río en Madre de Dios y la causa de la degradación de sus hábitats la minería aluvial, agricultura y ganadería cerca de los cauces de ríos. Esta situación sugiere la urgente necesidad del establecimiento de iniciativas de conservación en toda la cuenca del río Madre de Dios, en especial fuera de ANP.
\end{abstract}

Palabras clave: Madre de Dios; conservación; Amazonia; minería aluvial; especie amenazada; superdepredadores; poblaciones valuartes.

\section{Abstract}

Population status and geographic distribution of the giant otter (Pteronura brasiliensis) from Madre de Dios Region (south-eastern Peru) of 2014 and 2015 periods are presented. Population census for each period were 128 individuals, grouped in 22 and 20 resident groups during 2014 and 2015 periods respectively. The Manu National Park $(2.2 \mathrm{ind} / 10 \mathrm{~km})$ and Bahuaja Sonene National Park $(1.43 \mathrm{ind} / 10 \mathrm{~km}$.) were the sectors with the greatest abundance of giant otters inside the natural protected areas (ANP), while, Las Piedras and Los Amigos rivers were the sectors, outside the ANP, with highest value of abundance: 0.5 and $0.43 \mathrm{ind} / 10 \mathrm{~km}$ respectively. The populations inside ANP show more stable and protected (17 resident groups), while outside ANP are more vulnerable, where we registered only five (2014) and two (2015) giant otter groups. Currently, the main threats for giant other populations from Madre de Dios and the cause of the environmental degradation of its habitats are the gold mining, agriculture and logging. This situation suggests the urgent need to establish a conservation program throughout the region, especially outside ANP.

Keywords: Madre de Dios; conservation; Amazonia; Alluvial mining; endangered species; apex predator; Population stronghold.

Citación:

Mendoza J.A., K. Huamani, G. Sebastián y J.A. Ochoa. 2017. Distribución y estado poblacional de lobo de río (Pteronura brasiliensis) en la cuenca del río Madre de Dios, sureste del Perú. Revista peruana de biología 24(2): 155 - 162 (Julio 2017). doi: http://dx.doi.org/10.15381/ rpb.v24i2.13493

Presentado: $\quad$ 28/11/2016

Aceptado: $\quad 29 / 05 / 2017$

Publicado online: $20 / 07 / 2017$
Información sobre los autores:

JAM, KHV, GSD y JAO participaron en la recolección de datos y trabajos en campo; JAM análisis de los datos; JAM y JAO realizaron la redacción del manuscrito.

Los autores no incurren en conflictos de intereses.

Fuentes de financiamiento: Frankfurt Zoological Society-Perú; ICCA II-USAID 


\section{Introducción}

El lobo de río (Pteronura brasiliensis) es la nutría más grande de las 13 nutrias que actualmente existen a nivel mundial. Es una de las especies más amenazadas de la región amazónica, considerada en peligro de extinción según la Lista Roja IUCN (Groenendijk et al. 2015) y también se encuentra en el Apéndice I de CITES. En el Perú está considerado en situación de peligro (EN) y está protegido por el Estado Peruano mediante DS $\mathrm{N}^{\circ}$ 004-2014-MINAGRI. Esta especie ha perdido drásticamente su área de distribución en América del Sur y está limitada a áreas remotas o protegidas (Brack 1978, Carter \& Rosas 1997). En el Perú, $P$. brasiliensis estuvo a punto de extinguirse debido a cacería ilegal en la década de los 70, pero gracias al establecimiento de algunas áreas naturales protegidas (ANP) en la Amazonía y al control de la cacería en nuestro país, sus poblaciones han podido iniciar un proceso de recuperación que probablemente continúe en el presente (Carter \& Rosas 1997, Davenport 2008, Groenendijk \& Hajek 2006, Groenendijk et al. 2014). En el sureste del Perú, se encuentran poblaciones relativamente en buen estado dentro de ANP, como el Parque Nacional del Manu, la Reserva Nacional Tambopata y el Parque Nacional Bahuaja Sonene (Schenck 1999, Groenendijk et al. 2014) no obstante, las poblaciones de lobos de río fuera de ANP están sufriendo disminuciones preocupantes producto de la destrucción, degradación y fragmentación del hábitat principalmente producido por la minería aluvial que están poniendo en riesgo incluso las mismas poblaciones al interior de las ANP.

El hábitat del lobo de río son los cuerpos de agua como ríos, quebradas, pantanos y especialmente los lagos amazónicos (cochas); este último siendo el hábitat preferido donde se reproducen. Su presencia es sinónimo de buena calidad del agua por lo que han sido considerados como buenos indicadores de perturbaciones ecológicas y del estado de conservación de un lugar (Schenck 1999, Staib 2005). Si bien existen esfuerzos importantes de conservación dentro de ANP, ninguna unidad de conservación garantiza por si sola una población sostenible a largo plazo. Al ser una especie dependiente de un hábitat particular (cochas), la peculiaridad de su hábitat y requerimientos ecológicos y de comportamiento de vivir en grupos familiares, sumado a la pérdida y fragmentación de hábitat que viene ocurriendo fuera de las ANP, la convierten en una especie altamente vulnerable, reduciendo su potencial para la dispersión y colonización (de mucha importancia en lobo de río), subdividiendo las poblaciones en metapoblaciones que podrían impedir la conectividad genética y contribuyendo a extinciones locales.

El objetivo del presente estudio es dar a conocer la situación actual del lobo de río en la región de Madre de Dios a nivel poblacional y de distribución geográfica, que puedan ser de utilidad para el establecimiento de medidas conservación de esta importante especie. El presente estudio incluye los resultados de evaluaciones en la mayor parte de los afluentes de la cuenca del río Madre de Dios durante los ańos 2014 y 2015.

\section{Material y métodos}

Área de estudio.- El estudio fue realizado en la cuenca del río Madre de Dios localizada en el sureste del Perú (Fig. 1). El área de evaluación comprende siete sectores dentro de ANP del Parque Nacional del Manu (río Manu), Reserva Comunal Amarakaeri (ríos Blanco y Shilibe), Reserva Nacional Tambopata (cocha Sandoval, ríos La Torre y Tambopata) y el Parque Nacional Bahuaja Sonene (ríos Heath y Palma Real). Adicionalmente se evaluaron tres sectores fuera de las ANP que comprenden los ríos Los Amigos, Las Piedras y Tahuamanu. En todos los sectores se evaluaron los afluentes principales, quebradas y cochas.

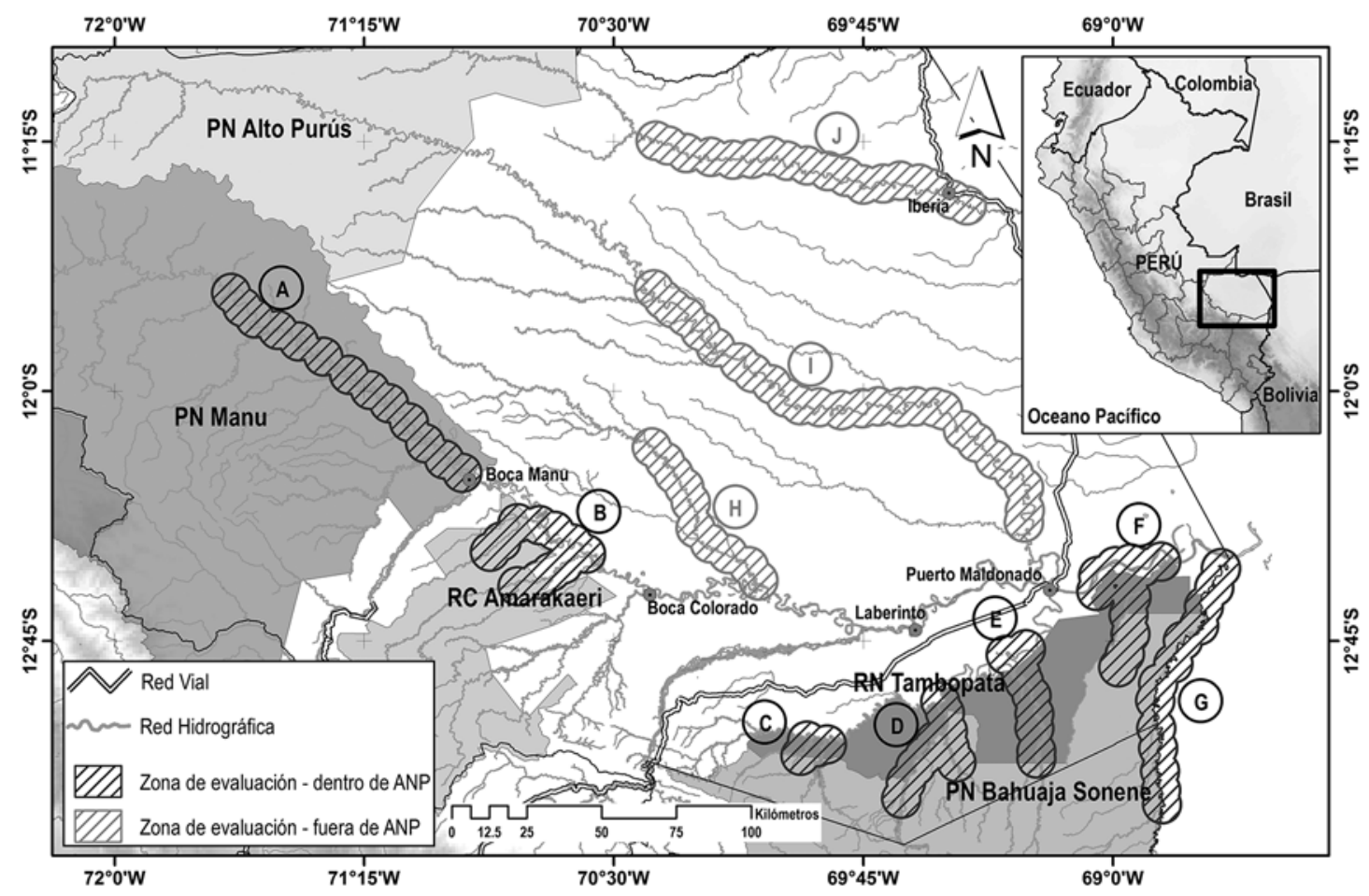

Figura 1: Afluentes ubicados dentro de ANP: A) Río Manu, B) Ríos Blanco y Shilive, C) Río Azul, D) Río Chuncho, E) Ríos La Torre y Tambopata, F) Río Palma Real y Cocha Sandoval, y G) Río Heath. Afluentes ubicados fuera de ANP: H) Río Los Amigos, I) Río Las Piedras y J) Río Tahuamanu. 
Tabla 1: Detalle de los ríos evaluados dentro y fuera de ANP en la región Madre Dios.

\begin{tabular}{|c|c|c|c|c|c|c|}
\hline & & $\begin{array}{l}\text { Cuerpos } \\
\text { de agua } \\
\text { evaluados }\end{array}$ & Cochas & $\begin{array}{l}\text { Longitud de } \\
\text { río recorrido } \\
(\mathrm{km})\end{array}$ & $\begin{array}{l}\text { Cochas } \\
\text { por } 10 \mathrm{~km}\end{array}$ & $\begin{array}{l}\text { Promedio del } \\
\text { área de cochas } \\
\text { (ha) }\end{array}$ \\
\hline \multirow{3}{*}{ Fuera de ANP } & Río Tahuamanu & 20 & 20 & 228.67 & 0.87 & 3.03 \\
\hline & Río Las Piedras & 12 & 11 & 309.25 & 0.36 & 10.00 \\
\hline & Río Los Amigos & 18 & 17 & 116.32 & 1.46 & 4.57 \\
\hline PN Manu & Río Manu & 17 & 17 & 179.94 & 0.94 & 22.76 \\
\hline \multirow{2}{*}{ RC Amarakaeri } & Ríos Blanco y Madre de Dios & 4 & 3 & 45.52 & 0.66 & 16.76 \\
\hline & Río Shilibe & 1 & 0 & 61.97 & 0.00 & 0.00 \\
\hline \multirow{6}{*}{$\begin{array}{l}\text { PN Bahuaja } \\
\text { Sonene y RN } \\
\text { Tambopata }\end{array}$} & Río Azul y Malinowski & 10 & 9 & 115.93 & 0.78 & 1.75 \\
\hline & Río Chuncho & 10 & 9 & 36.41 & 2.47 & 1.79 \\
\hline & Río La Torre & 9 & 8 & 68.13 & 1.17 & 2.39 \\
\hline & Río Tambopata & 2 & 2 & 113.04 & 0.18 & 58.30 \\
\hline & Río Palma Real y cocha Sandoval & 11 & 9 & 103.95 & 0.87 & 16.17 \\
\hline & Río Heath & 16 & 14 & 182.27 & 0.77 & 8.83 \\
\hline
\end{tabular}

Se evaluaron un total de 130 cuerpos de agua, entre cochas y quebradas, 80 de las cuales se ubican dentro de ANP (61.54\%). Se recorrieron aproximadamente $1561.4 \mathrm{~km}$ de longitud de ríos, siendo los mayores recorridos de evaluación en los ríos Heath $(182.27 \mathrm{~km})$, Manu $(179.94 \mathrm{~km})$, Las Piedras (309.25 $\mathrm{km})$ y Tahuamanu $(228.67 \mathrm{~km})$, asimismo los ríos con menor recorrido fueron los ríos Chuncho $(36.41 \mathrm{~km})$ y Blanco $(45.52$ $\mathrm{km})$ (Tabla 1).

Los ríos Chuncho y La Torre son los que presentan mayor abundancia de cochas dentro de ANP (2.47 cochas/10 km y 1.17 cochas $/ 10 \mathrm{Km}$ respectivamente, en caso de los afluentes ubicados fuera de ANP los ríos que presentaron una mayor abundancia de cochas son el río Los Amigos con 1.46 cochas/ 10 $\mathrm{km}$ y el río Tahuamanu con 0.87 cochas $/ 10 \mathrm{~km}$ (Tabla 1 ). Los mayores promedios de área del espejo de agua se encuentran en los ríos Tambopata (58.30 ha) y Manu (22.76 ha).

Evaluaciones.- Las evaluaciones se realizaron entre los meses de marzo a octubre de cada año (2014 y 2015). La metodología aplicada para este estudio fue la propuesta por Groenendjink et al. (2005), la cual se basa principalmente en:

Censo poblacional.- Conteo de todos los individuos por grupo observado en cada cuerpo de agua. Cuando se observó más de un grupo (o solitario) dentro de una cocha, la metodología establece que se separe el número correspondiente a cada uno con el signo + (más). De esta forma se indica la presencia de más de un grupo y el número de sus miembros (ejemplo: $8+1$ : ocho individuos más un solitario).

Uso de hábitat.- Se registraron todos los indicios de presencia de los lobos de río en cada uno de los cuerpos de agua:

- Campamentos: manchas irregulares de tierra, generalmente localizadas al lado de un cuerpo de agua, que han sido limpiadas por los lobos y que son usadas para defecación, marcación, secado, acicalamiento y descanso (Fig. 2).

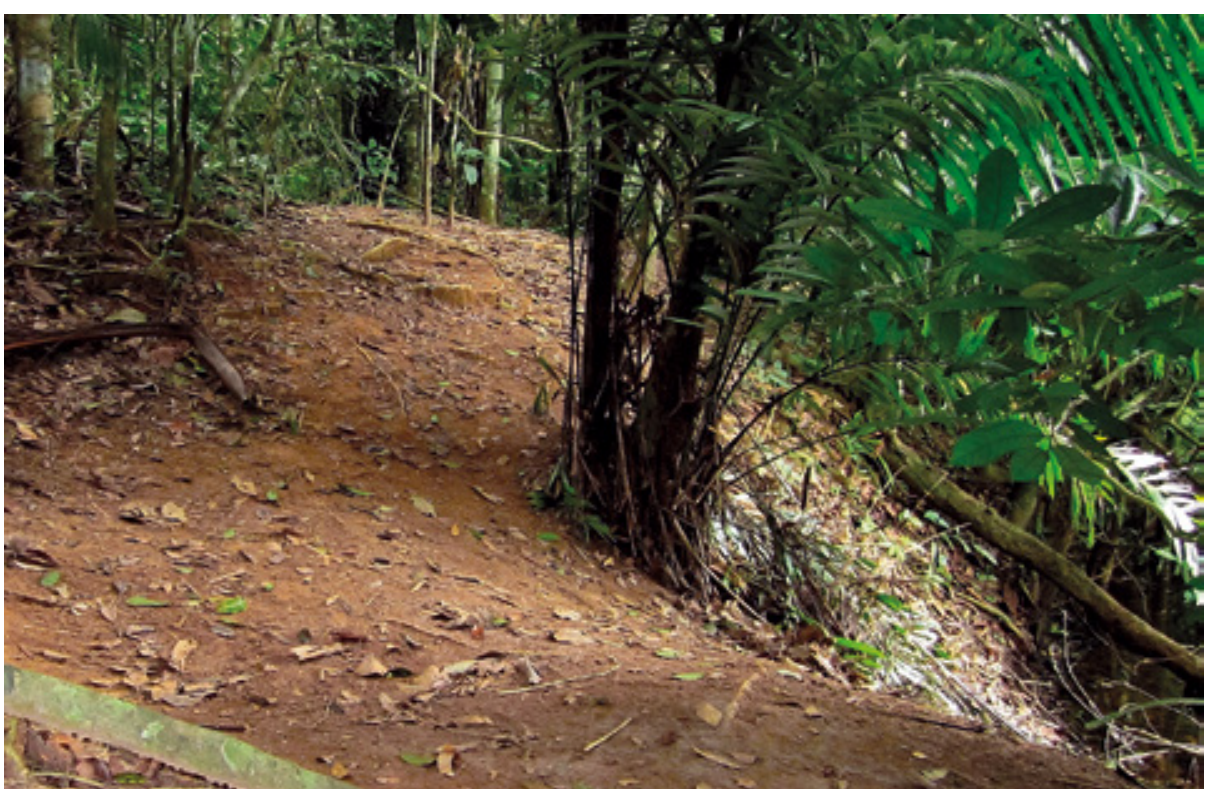

Figura 2. Campamento de lobo de río en el PN Bahuaja Sonene, cocha Sabaluyo. 


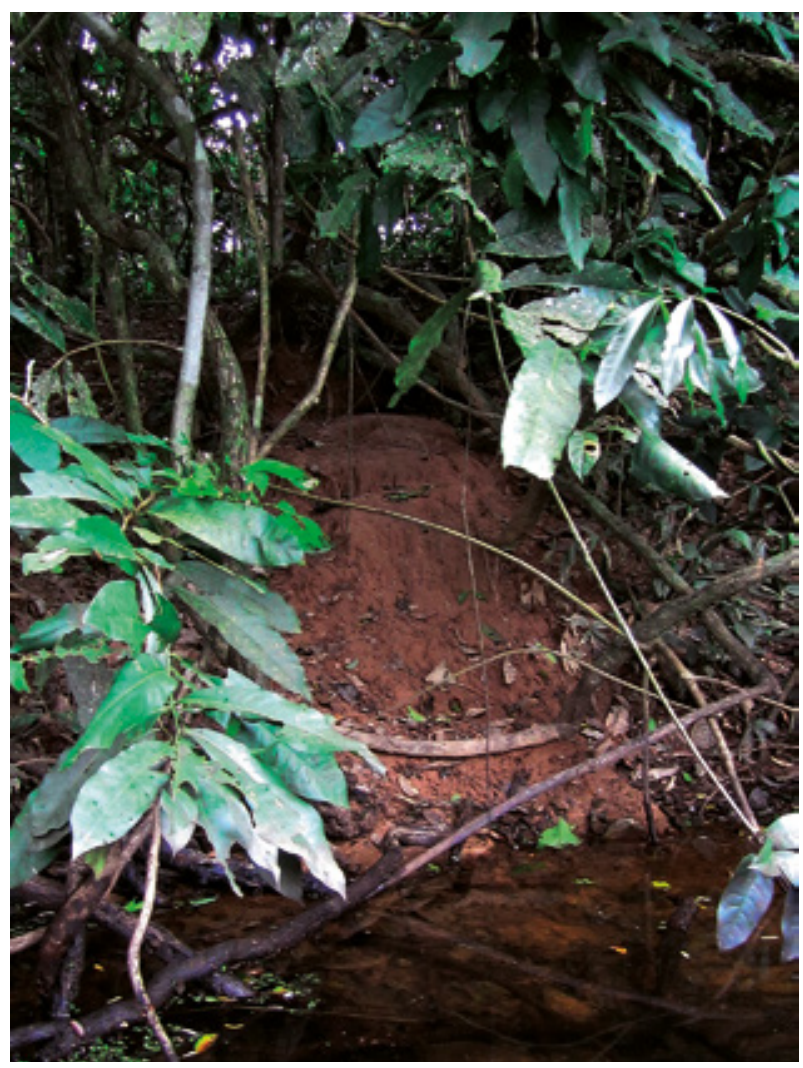

Figura 3. Madriguera de lobo de río en la RN Tambopata, río Azul.

- Madrigueras: consisten en uno o más túneles que se dirigen hacia una o más cámaras ovaladas que han sido escavadas en las orillas de un cuerpo de agua. Son usadas para dormir y para el cuidado de las crías. Generalmente existen campamentos cercanos (Fig. 3).

- Huellas: Son parecidas a las marcas dejadas por las puntas de los dedos humanos. Sólo se registraron cuando no estaban asociadas a ninguna de las evidencias anteriores.

\section{Resultados}

Registros de avistamiento e indicios de la presencia de lobo de río.- En el año 2014 el 46.92\% del total de los cuerpos de agua evaluados presentaban algún indicio de la presencia de lobo de río (campamentos y madrigueras); mientras que el $21.54 \%$ de los cuerpos de agua tuvieron registros directos de individuos de lobo de río. En el 2015 el registro de indicios bajo a 33.85\%, mientras que número de cuerpos de agua en donde se observó a la especie se incrementó a 23.08\% (Fig. 4)

Los mayores porcentajes de cuerpos de agua registrados con algún indicio de presencia de lobo de río en ambos periodos de evaluación estuvieron dentro de las ANP (31.54\% y 30.00\% respectivamente), lo mismo ocurre con las cochas donde se observaron a la especie. En el caso de los cuerpos de agua ubicados fuera de las ANP hubo un descenso en el número de cuerpos de agua que presentaron algún indicio de la presencia de lobo de río ya que para el año 2014 fue de $15.39 \%$ y para el 2015 bajó a 3.85\% (Fig. 4).

Censo de lobo de río.- En cada uno de los periodos de evaluación se registraron 128 lobos de río, siendo los ríos Manu y Heath, ubicados en el PN Manu y en el PN Bahuaja Sonene respectivamente y el río Las Piedras ubicado fuera de las ANP, las cuencas que presentan las mayores poblaciones de lobo de río (Fig. 5). Por el contrario el río Shilive ubicado en la RC Amarakaeri y el río Tahuamanu, tuvieron los menores registros, a lo sumo escasos indicios de su presencia, suponiendo que sus poblaciones son muy escasas en estos sectores.

De los 128 individuos registrados durante la evaluación del 2014, 106 residían dentro de las ANP, estos estuvieron distribuidos en 17 grupos familiares, una pareja y 6 solitarios (o dispersores), siendo el río Manu donde se encontraron el mayor número de grupos familiares (Tabla 2). Los mayores grupos familiares observados estuvieron integrados por 8 individuos, que se ubicaron en los río Manu y Heath (Fig. 6); fuera de las ANP se registraron 22 lobos de río los que estaban distribuidos en 5 grupos familiares y dos solitarios, el mayor grupo familiar fue de 6 individuos ubicado en el río Las Piedras.

En la evaluación del año 2015, se registraron 114 individuos dentro de las ANP, distribuidos en 18 grupos familiares, 5 parejas y 4 solitarios; fuera de ANP se observó a 14 individuos distribuidos en 2 grupos familiares y 2 parejas de lobos de río, todos estos registrados en el río Las Piedras (Tabla 2). En esta última evaluación los mayores grupos familiares fueron nuevamente registrados en el río Manu, con un grupo familiar de 15 individuos y en el río Heath el mayor grupo fue de 9 individuos.

Distribución.- Los lobos de río se distribuyen en toda la región de Madre de Dios (Fig. 8), concentrándose principalemnet en las ANP. La especie se encuentra totalmente ausente en el río Inambari debido principalmente a la fuerte presencia de actividades extractivistas de los recursos alrededor de este afluente (principalmente minería aluvial), ocasionando el deterioro y alteración de los cuerpos de agua (obs. pers.), como puede observarse en la Figura 7, esto se evidencia también en gran parte del río Madre de Dios, río Malinowski por lo que los

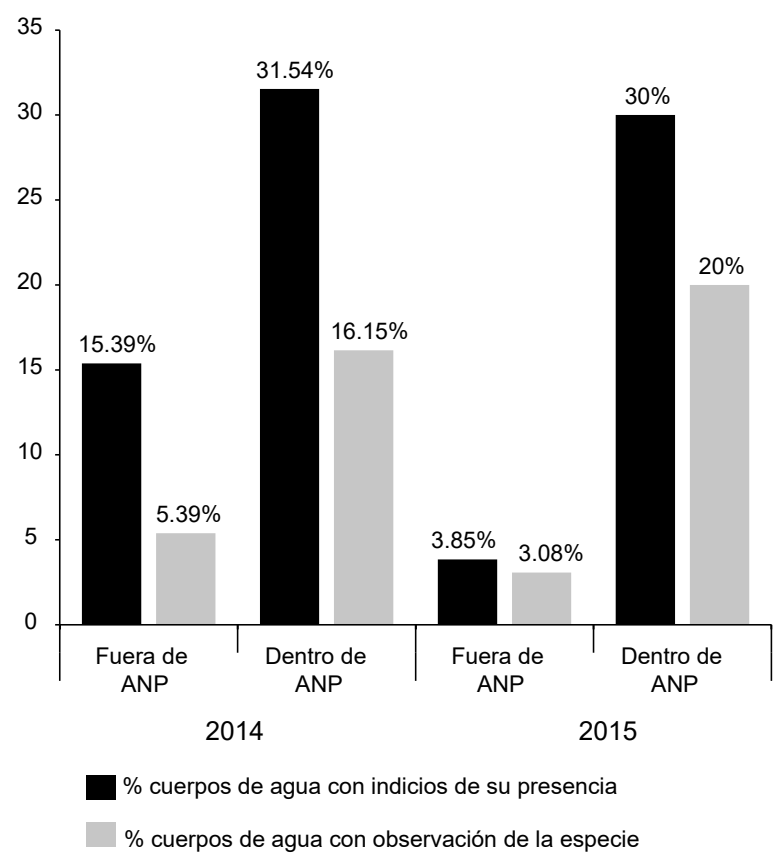

Figura 4: Porcentaje de cochas donde se avistaron y registraron indicios de la presencia de lobo de río en los años 2014 y 2015. 

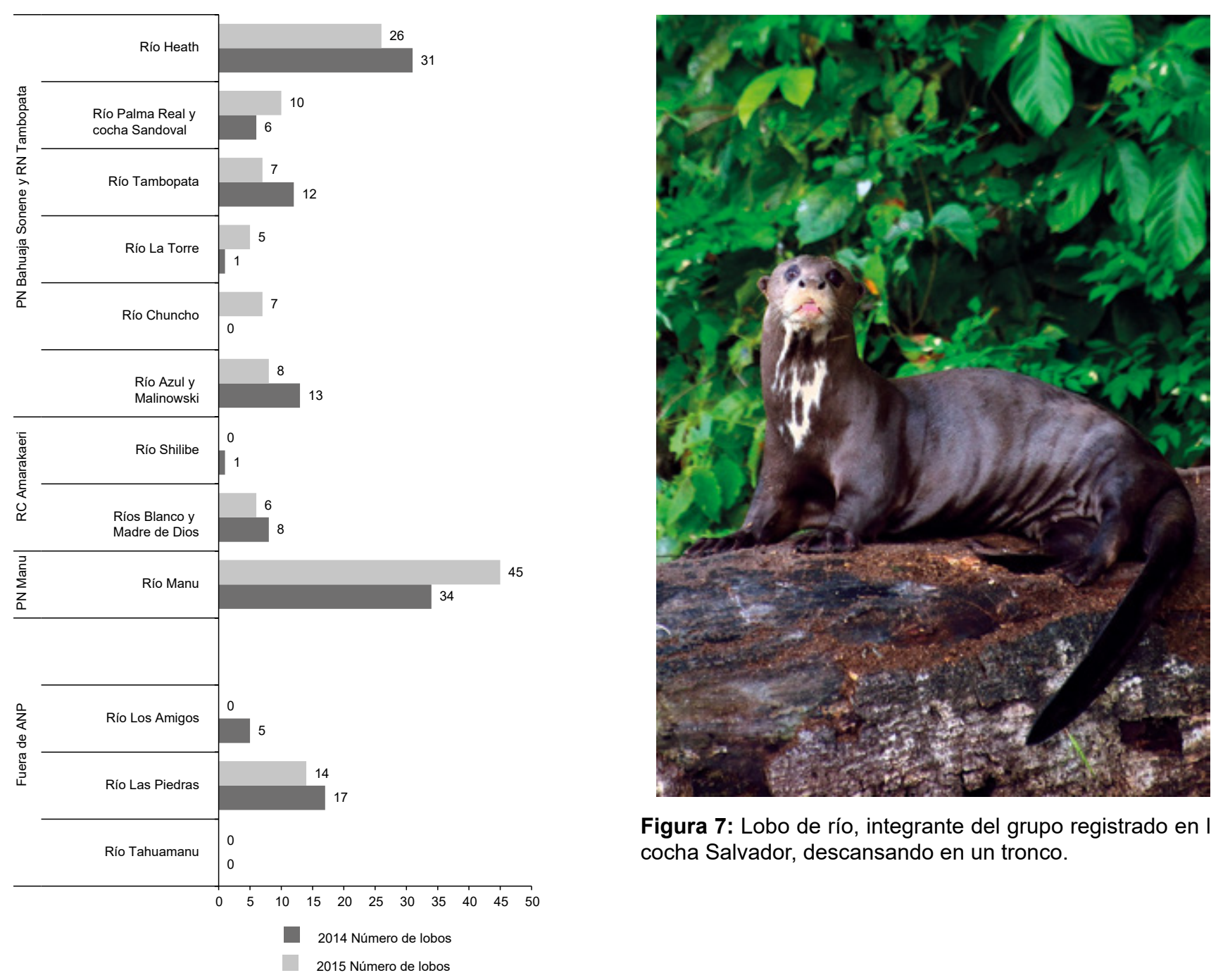

Figura 7: Lobo de río, integrante del grupo registrado en la cocha Salvador, descansando en un tronco.

Figura 5: Avistamientos de lobo de río en los diferentes sectores de la cuenca del río Madre de Dios para los años 2014 y 2015
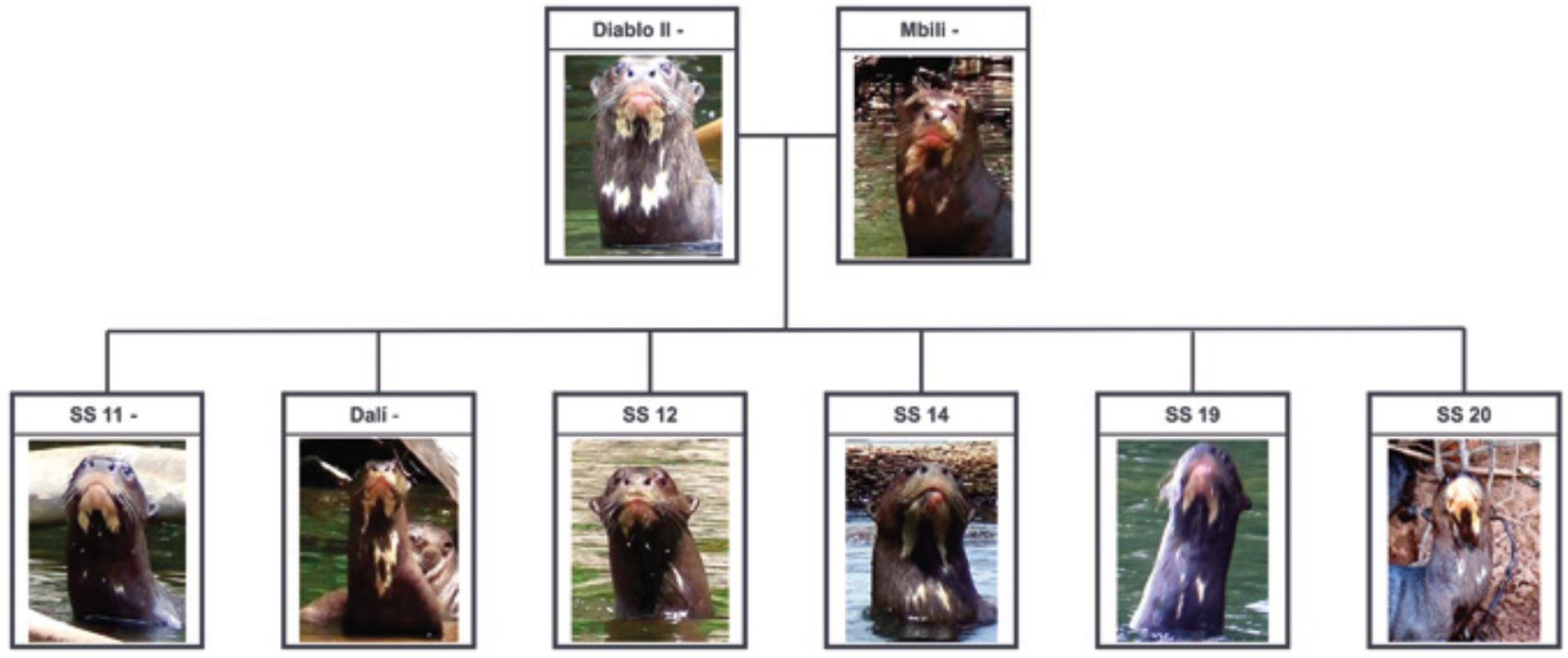

Figura 6: Grupo familiar registrado en la cocha Salvador en el PN Manu durante la evaluación del 2014. 
Tabla 2: Número de grupos familiares (indicando el número de individuos por cada grupo), parejas y solitarios registrados en ambos periodos de evaluación en la región Madre de Dios.

\begin{tabular}{|c|c|c|c|c|c|c|c|}
\hline & & \multicolumn{3}{|c|}{ Evaluación - 2014} & \multicolumn{3}{|c|}{ Evaluación - 2015} \\
\hline & & $\begin{array}{c}\text { Grupos } \\
\text { familiares }\end{array}$ & $\begin{array}{c}\mathrm{N}^{\circ} \text { de } \\
\text { pares de } \\
\text { individuos }\end{array}$ & $\begin{array}{c}\mathbf{N}^{\circ} \text { de } \\
\text { solitarios }\end{array}$ & $\begin{array}{c}\text { Grupos } \\
\text { familiares }\end{array}$ & $\begin{array}{c}\mathrm{N}^{\circ} \mathrm{de} \\
\text { pares de } \\
\text { individuos }\end{array}$ & $\begin{array}{c}\mathbf{N}^{\circ} \text { de } \\
\text { solitarios }\end{array}$ \\
\hline \multirow{3}{*}{ Fuera de ANP } & Río Tahuamanu & 0 & 0 & 0 & - & - & - \\
\hline & Río Las Piedras & $4+4+3+5$ & 0 & 1 & $4+6$ & 2 & 0 \\
\hline & Río Los Amigos & 4 & 0 & 1 & - & - & - \\
\hline PN Manu & Río Manu & $\begin{array}{c}6+7+4+8 \\
+3+4\end{array}$ & 1 & 0 & $\begin{array}{l}4+6+3+3 \\
+15+5+4 \\
\end{array}$ & 2 & 1 \\
\hline \multirow{2}{*}{ RC Amarakaeri } & Río Blanco y Río Madre de Dios & $5+3$ & 0 & 0 & 4 & 1 & 0 \\
\hline & Río Shilibe & 0 & 0 & 1 & - & - & - \\
\hline \multirow{6}{*}{$\begin{array}{l}\text { PN Bahuaja } \\
\text { Sonene y RN } \\
\text { Tambopata }\end{array}$} & Río Heath & $6+8+8+5$ & 0 & 4 & $3+7+9+5$ & 0 & 2 \\
\hline & Río Tambopata & $7+5$ & 0 & 0 & 5 & 1 & 0 \\
\hline & Río La Torre & 0 & 0 & 1 & 5 & 0 & 0 \\
\hline & Río Palma Real y Cocha Sandoval & 6 & 0 & 0 & $7+3$ & 0 & 0 \\
\hline & Río Azul y Malinowski & $8+5$ & 0 & 0 & 7 & 0 & 1 \\
\hline & Río Chuncho & - & - & - & 5 & 1 & 0 \\
\hline
\end{tabular}
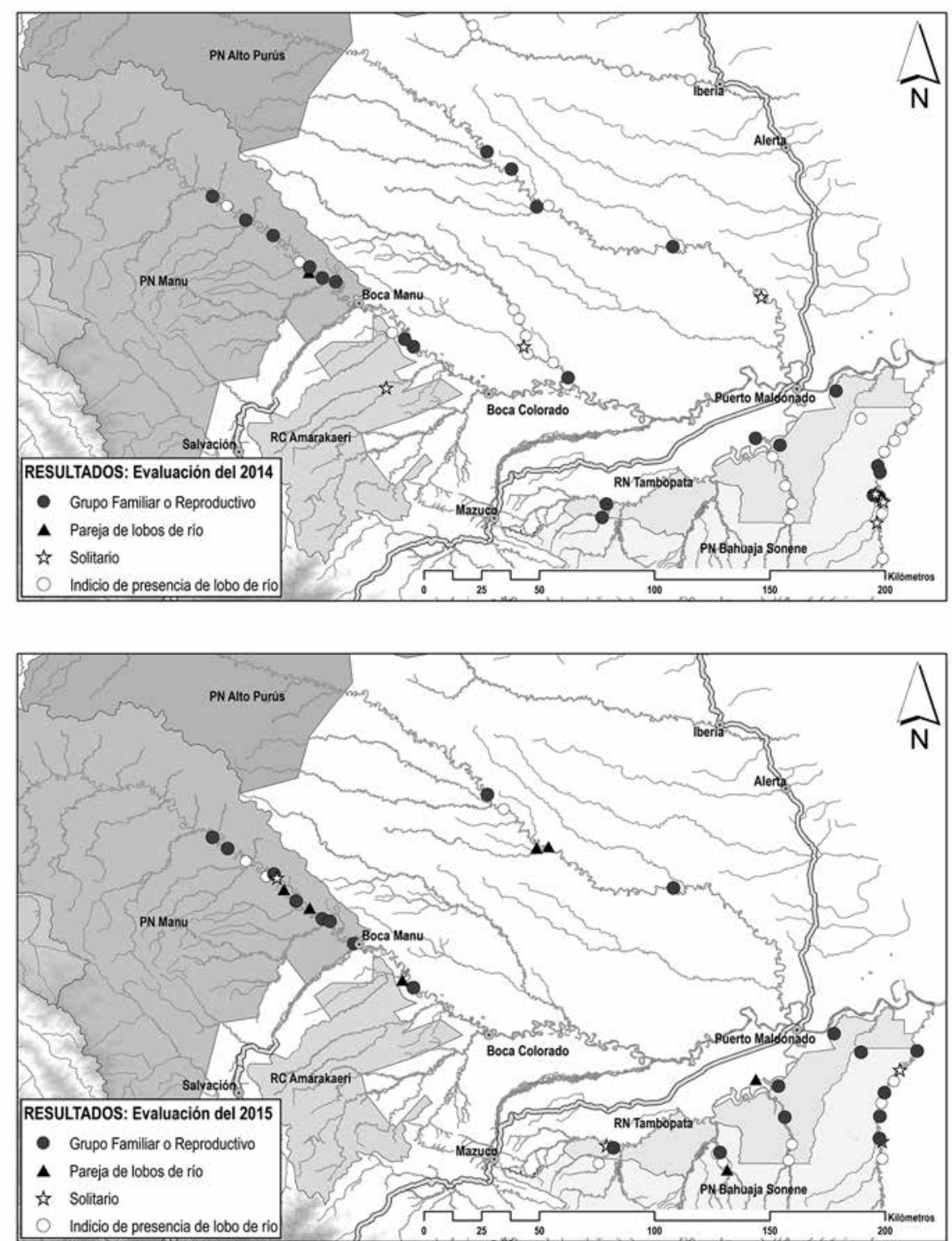

Figura 8: Distribución de los avistamiento de lobo de río en cada uno de los periodos de evaluación en la cuenca del Madre de Dios. 


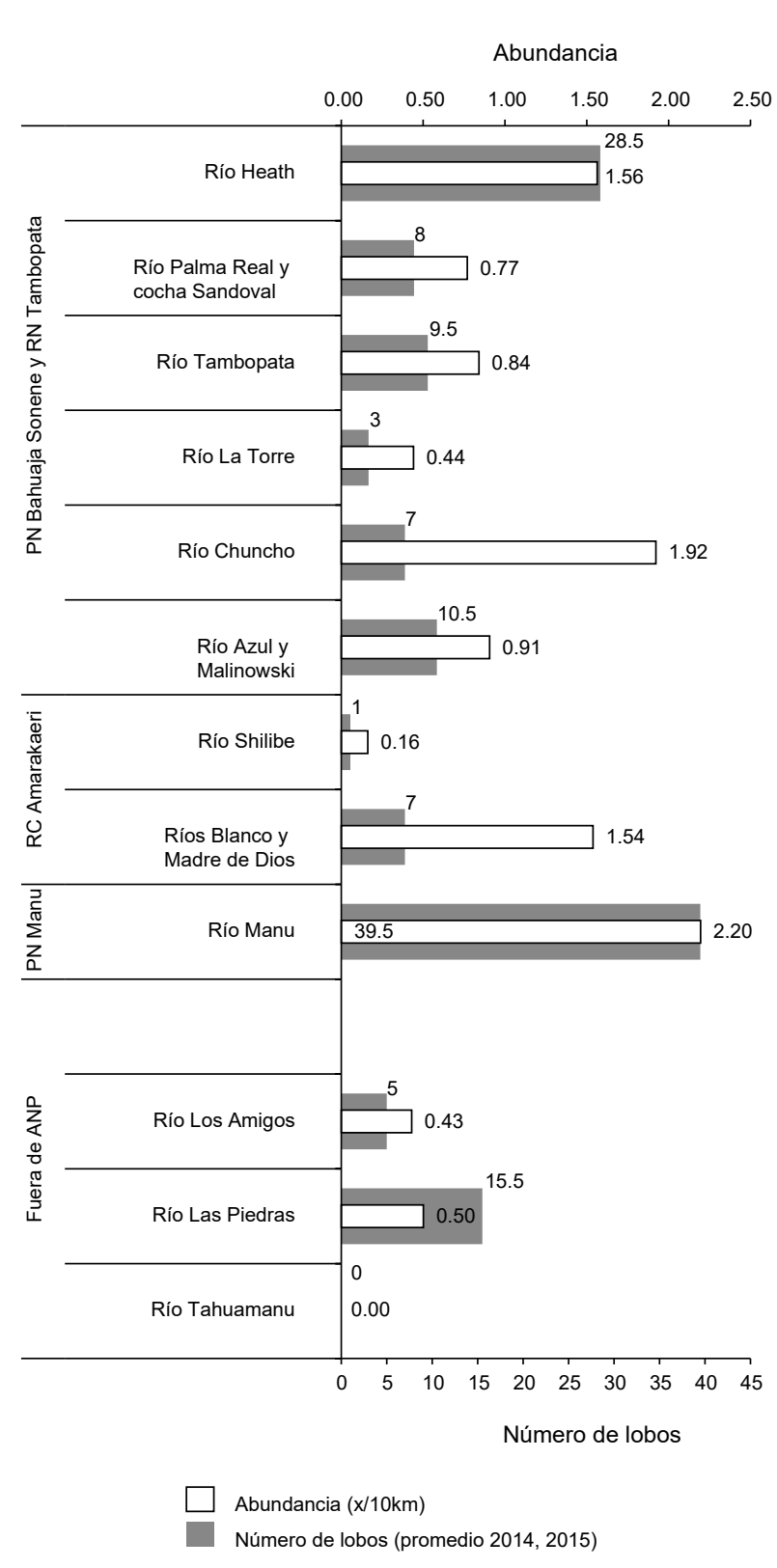

Figura 9: Promedio del número de lobos de río registrados en ambos épocas de evaluación y la Abundancia obtenida en cada una de las zonas evaluadas.

avistamientos de lobo de río solo se registran en zonas donde se realiza la actividad turística o donde existe un puesto de control y vigilancia de algún ANP.

En el caso del río Tahuamanu no se pudo avistar a la especie pero se registraron indicios de su presencia, por lo que no podemos afirmar que el lobo de río se encuentre totalmente erradicado de esta zona. Suponiendo que el número de lobos de río es bastante escasa.

Aspecto poblacional.- Las mayores poblaciones de lobo de río están presentes en los río Manu (39.5 individuos) y Heath (28.5 individuos) con una abundancia de 2.20 individuos $/ 10 \mathrm{~km}$ y 1.56 individuos $/ 10 \mathrm{~km}$ respectivamente, ubicadas dentro de ANP, en el caso de los afluentes ubicados fuera de ANP se tiene que el río Las Piedras alberga la mayor población de la especie con una abundancia aproximada de 0.50 individuos $/ 10 \mathrm{~km}$ (Fig. 9).

\section{Discusión}

Los lobos de río en la región Madre de Dios prefieren habitar las cochas de manera preferente en lugar de quebradas y ríos, por lo que el tamaño y frecuencia con que se presentan las cochas, meandros abandonados, son determinantes. La población de lobos de río en Madre de Dios sigue siendo pequeña (128 individuos). Las mayores poblaciones de lobo de río se encuentran dentro de ANP constituyéndose en los principales refugios así como importantes poblaciones sumideros que mantendrá a las demás poblaciones o subpoblaciones.

Entre los principales ríos de la región Madre de Dios, el Manu y Heath, son los adecuados para los lobos de río, porque presentan un alto número de cochas (17 y 14 cochas respectivamente) y un área importante de espejo de agua, por lo que albergan las mayores poblaciones de la especie la región. Los ríos Azul, Los Amigos y Tahuamanu presentan una alta abundancia de cochas, pero estos cuerpos de agua son más pequeños por lo que la presencia de lobos de río es bastante reducido en comparación a los demás ríos. En el caso del río Madre de Dios, esta presenta las condiciones naturales más apropiadas (grandes cochas y una relativa alta abundancia), pero la baja presencia de lobos de río es explicado por la alta actividad humana que se observa a lo largo del cauce del río (Schenck 1999, Groenendijk et al. 2006, Davenport 2008)

La distribución de los grupos familiares en todo el ámbito de estudio no es uniforme, en muchos lugares no se registró la presencia de lobo de río aunque presenten condiciones adecuadas, por lo que la especie se encuentra aún en un proceso de recuperación dentro de la cuenca del río Madre de Dios (Groenendijk et al. 2014).

El río Manu es el sector que presenta la mayor población de lobos de río (2.2 individuos/10 km) en la región y está ubicada dentro de un ANP y el sector fuera de ANP con la mayor población de lobos de río es el río las Piedras con 0.50 individuos $/ 10 \mathrm{~km}$. Estos datos obtenidos en comparación a los obtenidos por Flores (2012) de la Zona Reservada de Güeppí, en los ríos Peyena y Angustilla (norte del Perú) presentan leves diferencias a las obtenidas en el presente estudio, siendo las abundancias obtenidas de 2.90 individuos $/ 10 \mathrm{~km}$ y 2.33 individuos $/ 10 \mathrm{~km}$ respectivamente. Por otro lado en Brasil se calculó una abundancia de 5.67 individuos $/ 10 \mathrm{~km}$ en el río Vermelho (Leuchtenberger \& Mourão 2008) y en Bolivia en el río Paragua se calculó una abundancia de 15.60 individuos $/ 10 \mathrm{~km}$ (Van Damme et al. 2002), estos datos son mayores a los obtenidos en Madre de Dios, pero probablemente se deba a que en estos ríos tienen una mayor disponibilidad de hábitat o una mayor protección o aislamiento (Ayala \& Wallace 2009).

Los resultados de este estudio sugieren que la cuenca del río Madre de Dios, posee una escasa población de lobos de río, y que presenta una situación crítica debido a que están expuestos a varias amenazas, principalmente la minería aluvial y otras actividades antropogénicas como a agricultura, ganadería y la tala de madera. La minería en Madre de Dios se ha incrementado notablemente en los últimos ańos, y la presencia de lobo de río en ríos como Malinowski e Inambari es inexistente. La minería ha ocasionado la perdida y contaminación de los ecosistemas acuáticos afectando así a la calidad y cantidad de hábitats saludables y disponibles para la especie. Es necesario desarrollar e 
implementar un plan de acción para la conservación del lobo de río, principalmente creando nuevas áreas de protección para la especie (especialmente fuera de ANP) y promover la creación de corredores acuáticos entre las poblaciones registradas buscando reducir el aislamiento de estas poblaciones.

Finalmente es importante resaltar que el lobo de río se constituye como uno de los principales atractivos para el turismo en Madre de Dios y una importante fuente de ingresos para la población local, por lo que ha sido declarada como especie representativa de Madre de Dios por Ordenanza Regional (OR $\mathrm{N}^{\circ}$ 005-2011-GRMDD/CD). En ese entender la promoción de iniciativa de turismo sostenible en la región Madre de Dios puede ser una de las principales estrategias para la conservación del lobo de río.

\section{Agradecimientos}

Al Programa de "Conservación de lobo de río" financiado por Frankfurt Zoological Society-Perú, al proyecto "Conservado las cabeceras del corredor de Conservación Purús-Manu”, financiado por ICCA II-USAID y al proyecto "Conservación de Lobo de Río (Pteronura brasiliensis) en la región de Madre de Dios" financiado por el Consorcio Loreto Manu Tambopata de ICAA - USAID, que permitió realizar las evaluaciones poblacionales de lobo de río en gran parte de la cuenca del río Madre de Dios. Asimismo agradecer a las jefaturas y especialistas del SERNANP (Servicio Nacional de Áreas Naturales Protegidas por el Estado Peruano) del Parque Nacional del Manu, Parque Nacional Bahuaja Sonene, Reserva Nacional Tambopata y Reserva Comunal Amarakaeri por facilitar por los permisos correspondientes para realizar el presente estudio y la logística en los trabajos de campo, y principalmente a todos compañeros y voluntarios de la FZS y guardaparques del SERNANP que apoyaron durante las evaluaciones de campo.

\section{Literatura Citada}

Ayala G. \& R. Wallace. 2009. Distribución y abundancia de la Lontra (Pteronura brasiliensis) en Alto Madidi, Parque Nacional Madidi, La Paz, Bolivia. Revista Boliviana de Ecología y Conservación Ambiental 25. pp. 41-49.

Brack A. 1978. Situación actual de las nutrias (Lutrinae, Mustelidae) en el Perú. En Duplaix, N. (ed), Proceedings First Working Meeting of the Otter Specilist Group, Paramaribo, Suriname. Publication de la IUCN; pp. 76-84.

Carter S. \& F. Rosas. 1997. Biology and conservation of the Giant otter Pteronura brasiliensis. Mammal Review 27. pp. 1-26. http://dx.doi.org/10.1111/j.1365-2907.1997.tb00370.x

Davenport L. 2008. Behavior and Ecology of the Giant Otter (Pteronura brasiliensis) in oxbow lakes of the Manu Biosphere Reserve, Peru. Ph.D. Thesis. University of North Carolina. $245 \mathrm{pp}$.
DS No 004-2014-MINAGRI. 2014. Decreto Supremo que aprueba la actualización de la lista de clasificación y categorización de las especies amenazadas de fauna silvestre legalmente protegidas. El Peruano, Normas Legales, martes 8 de abril de 2014: 520497-520504

Flores F. 2012. Aspectos ecológicos y poblacionales de Pteronura brasiliensis (Zimmermann, 1780) "Lobo de Río", en la cuenca de los ríos Peneya y Angusilla, Zona Reservada de Güeppí. World Wildlife Fund, Perú. 66pp.

Groenendijk, J. 1998. Review of the Distribution and Conservation Status of the Giant Otter (Pteronura brasiliensis), with Special Emphasis on the Guyana Shield Region. Amsterdam: Netherlands Commitee for IUCN. 55pp.

Groenendijk, J., F. Hajek, N. Duplaix, C. Reuther, P. Van Damme, C. Schenck, E. Staib, R. Wallace, E. Waldemarin, R. Notin, M. Marmontel, F. Rosas, G.E. De Mattos, E. Evangelista, V. Utreras, G. Lasso, H. Jacques, K. Matos, I. Roopsind $\&$ J. Botello. 2005. Surveying and Monitoring Distribution and Population Trends of the Giant Otter (Pteronura brasiliensis) - Guidelines for a Standardization of Survey Methods as recommended by the Giant Otter Section of the UICN/SSC Otter Specialist Group. Habitat 16. Frankfurt Zoological Society. 104pp.

Groenendijk, J. \& F. Hajek. 2006. Lobos del río Madre de Dios. Ayuda para la Vida Silvestre Amenazada. Sociedad Zoológica de Fráncfort. Lima. 160 pp.

Groenendijk J., F. Hajek, P.J. Johnson, D.W. Macdonald, J. Calvimontes, E. Staib, y C. Schenck. 2014. Demography of the Giant Otter (Pteronura brasiliensis) in Manu National Park, South-Eastern Peru: Implications for Conservation. PLOS ONE 9 (8): e106202. doi:10.1371/journal.pone.0106202.

Groenendijk, J., N. Duplaix, M. Marmontel, P. Van Damme \& C. Schenck. 2015. Pteronura brasiliensis. The IUCN Red List of Threatened Species 2015. Acceso en línea en http://dx.doi.org/10.2305/IUCN.UK.2015-2.RLTS. T18711A21938411. en. Fecha de acceso 10/10/2016

Leuchtenberger, C. \& G. Mourão. 2008. Social Organization and Territoriality of Giant Otters (Carnivora: Mustelidae) in a Seasonally Flooded Savanna in Brazil. Sociobiology 52(2): $257-270$.

OR No 005-2011-GRMDD/CR- Declaran al Lobo de Río como especie representativa de la Región de Madre de Dios. El Peruano, Normas Legales, 15 de octubre de 2011: 451704- 451706

Schenck, C. 1999. Lobo de Río (Pteronura brasiliensis). Presencia, uso del hábitat y protección en el Perú. GTZ / INRENA. Lima, Perú. [Traducción al español de la Tesis Doctor, Universidad Ludwig-Maximilians. Munich], 176pp.

Staib, E. 2005. Eco-etología del Lobo de Río (Pteronura brasiliensis) en el Suroeste del Perú. Ayuda para la Vida Silvestre Amenazada - Sociedad Zoológica de Fráncfort. Lima - Perú. 195pp.

Van Damme, P., S. Ten, R. Wallace, L. Painter, A. Taber, R. Gomales, A. Fraser, D. Rumiz, C. Tapia, H. Michels, Y. Delaunoy, J. Saravia, J. Vargas \& L. Torres. 2002. Distribución y estado de las poblaciones de Lontra (Pteronura brasiliensis) en Bolivia. Revista Boliviana de Ecología 12. Pp. 111 - 134. 